\title{
The research of a modified estimate scheme for the satellite link level
}

\author{
Wang Xiao, He Qian, Zhu Yuming
}

China Satellite Maritime Tracking and Control Department, Jiangyin, 214431, China

\author{
Keywords: Satellite link, Level estimating, Precision, Automation
}

\begin{abstract}
The traditional estimate method adopted man-made calculation as for the satellite tracking and control link level. But this model was simple for the most of calculation was adopted the experience data, and did not think over the complicated influence factor. In order to do better on the estimate precision in the following tests, this paper puts a new optimization method of level estimating. Besides, we realized the. automation of calculate using the software. By this method, the post personnel's work load was reduced, and the precision for the level estimating was raised.
\end{abstract}

\section{Introduction}

Satellite ground station satellite ground refers to building link between satellite and ground monitoring stations and radar tracking effective data transmission path, you can use the time window between the satellite and ground stations and other parameters to describe. When the satellite is calculated for the effective monitoring and control ground station equipment Control window downlink channel theory margin world, confirm that the overall arc tracking and control equipment whether the target is always in cooperation within the effective antenna coverage usually required before performing the test, according to the Interface Control Document and the world cooperative target positions launch site test parameters provided in order to analyze the impact the capture phase tracking satellite downlink signal quality flight characteristics, a clear test of the arc focus CONTROL event.

Traditional links for satellite monitoring level estimating method using manual calculation and estimation model is relatively simple; the model used in the numerical mostly empirical data, the calculation process does not consider the impact of complex factors. And because the satellite platform and diverse, experimental models and complex situation, to estimate parameters of the different tests are different, the calculation complicated and error-prone process of calculating the level of changes in staff positions put forward higher requirements, and the introduction of artificial in the calculation process factors, which inevitably introduces more error factors that affect the level of estimation accuracy. Due to lack of level estimation process intelligence and procedures, not only increasing the workload of staff positions, which does not help to improve the accuracy of estimating the level, a lack of certainty as commanding officers throughout the trial level changes effective means. Therefore, factors influencing the level of estimation and try to raise the level of automation for software estimation accuracy of the actual implementation of the pilot have a positive meaning.

\section{Traditional level estimating method analysis}

Mathematical model uses the traditional method of computing segments, first calculate the ground control station at the entrance to the level of the size of the discharge, followed by a comprehensive base band received signal to noise ratio calculated according to $G / T$ value, calculated according to the empirical formula finally, specific Proceed as follows:

Usually for satellite downlink signal level according to the following basic formula:

$$
P_{s r}=E I R P-L_{s p}-L_{p}-L_{t e p}-L_{a t m}+G_{e r}
$$

Wherein, from left to right meaning of the parameters were put ground control station at the entrance level, flying cooperation objectives antenna aperture at the effective radiated power, 
electromagnetic waves in free space propagation loss, polarization loss, loss of pointing errors, atmospheric losses, ground antenna gain.

Free-space propagation loss, in accordance with the following formula:

$$
L_{s p}=20 \lg F+20 \lg R+32.44
$$

(2)

$\mathrm{F}$ is the operating frequency; $\mathrm{R}$ is the radial distance tracking.

Ground equipment for receiving satellite signals strength calculation formula:

$$
S / \Phi=E I R P+G / T-K-L_{s p}-L_{p}-L_{t e p}-L_{a t m}
$$

(3)

Wherein, $\mathrm{G} / \mathrm{T}$ antenna based quality factor, taking $22.5 \mathrm{~dB} / \mathrm{K} ; \mathrm{K}$ is Boltzmann's constant, taking $-228.6 \mathrm{dBW} / \mathrm{Hz}$.

The calculation result of the above drawing, as shown in Figure 1.

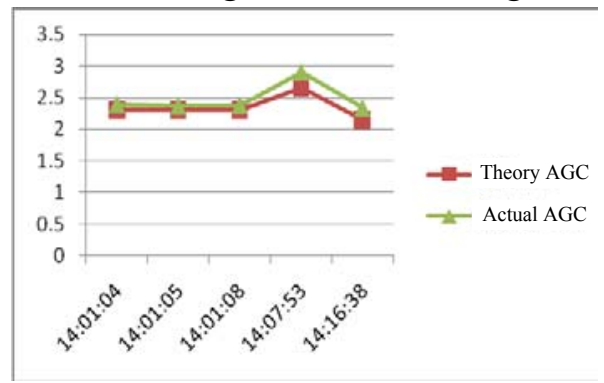

figure 1. Comparative tests of a theory with the actual AGC AGC

As can be seen by comparing the theoretical and actual values of the estimated level there are some errors in comparison, and this difference is maintained within a certain range, to minimize the error, the estimation accuracy further raise the level, it becomes a We need to study the issue.

\section{Raise the level of estimation accuracy Method}

From the satellite link design goal, the signal from the originating station to the terminal can be divided into: - satellite link control station (referred uplink); satellite; satellite - monitoring station (referred to as the downlink) three main components. The main factors which affect the monitoring stations to receive satellite downlink signal level are: the launch of the satellite EIRP, antenna gain ground, put field sensitivity, dynamic range, slant range and a variety of wear and tear.

a. Control group downlink antenna array pattern

According to the satellite flight attitude, the stars on the day of measurement and control angle with the ground control station to the antenna, combined with satellite monitoring group downlink antenna array pattern can be observed calculated for each target tracking station antenna tracking antenna net gain arcs, consider Spaceborne transmit power to the answering machine, feeder loss and power loss points to further cooperate to determine the value of the target relative measurement and control station EIRP.

Different fired and telemetry transmitter antenna mounted Arrow launch vehicle location, tracking ship cloth to launch its stations have different requirements, but also considering the position, course and other information factors during level estimates.

Also in the calculation process should also be considered rocket into tracking ship track after running arc attitude.

b. The signal to noise power spectral density calculation

According beacon radar equation formula and ground station monitoring and control system for target acquisition performance requirements, the system can be deduced monitoring stations to meet the requirements of the signal to noise power spectral density required is calculated as follows:

$$
S / \phi=E I R P G / T-K-(20 \mathrm{~g} F+20 \lg R+3244)-L_{p}-L_{\text {eep }}-L_{\text {atr }}
$$

Formulas: EIRP for the cooperation objectives antenna aperture at the effective radiated power; $\mathrm{G} / \mathrm{T}$ main antenna quality factor; $\mathrm{K}$ is Boltzmann's constant, $-228.6 \mathrm{dBW} / \mathrm{Hz}$; $\mathrm{F}$ for the device 
operating frequency; $\mathrm{R}$ radial tracking target distance; $\mathrm{L}_{\mathrm{p}}$ is polarization loss, $\mathrm{dB} ; \mathrm{L}_{\text {tep }}$ pointing error loss, $\mathrm{dB} ; \mathrm{L}_{\mathrm{atm}}$ atmospheric loss, $\mathrm{dB}$.

c. Calibration curves $A G C \sim S / \Phi$

In aerospace measurement and control system, downlink reception link $U_{A G C} \sim S / \phi$ curve has a very important role; it is one of the target angle to capture the process of "self-tracking" operating mode switching basis, but also the validity of the measured data track, tracking process analysis of abnormal phenomena important basis for judgment. Therefore, in the normal system installation, equipment work stable, requiring regular calibration curve $A G C \sim S / \Phi$ system, usually in the presence put before the directional coupler input port of the input frequency ordered dynamic level test in the downlink receive chain port access spectrum analyzer, read $S / \varphi$ value, while in the corresponding baseband interface integrated readout AGC voltage value. Finishing the above data and plotted as a curve $U_{A G C} \sim S$ / $\phi$, for the implementation of the pilot use. During the test, according to the size $U_{A G C}$ voltage level can be preliminary estimated value of the real-time reception of satellite signals. Combined with the actual situation calibration, the curve should satisfy a linear relationship.

d. Other Loss Analysis

Satellite link level estimation, in addition to signal transmission loss in free space, and some loss of accuracy must also be considered when calculating.

Feeder loss:

There is loss between the receiving antenna and the receiver itself, which appears in the connection waveguides, filters and couplers, and is represented by the receiver feeder loss. In fact, there are such high loss between the transmitting antenna and the amplifier output, this loss has been considered if given EIRP, do not repeat consideration.

Antenna misalignment loss:

Ideally, communication, monitoring and control station antennas and satellite antennas are aligned, then the maximum antenna gain. In fact, the situation is axially offset from the satellite antenna will often arise. Off-axis loss there are two possibilities: a satellite antenna, a control station antenna is shown in Figure 2. Appeared on the satellite off-axis loss with a gain cross-sectional view of the actual satellite antenna design (satellite antenna patterns), control station antenna gain axial deviation leads to decline, known as point losses, usually in a few tenths to a few little between decibels, no big antenna tracking device, point losses may be large. Polarization direction misalignment antenna feed, may also have losses, but the value is generally small, often using statistical data to estimate.
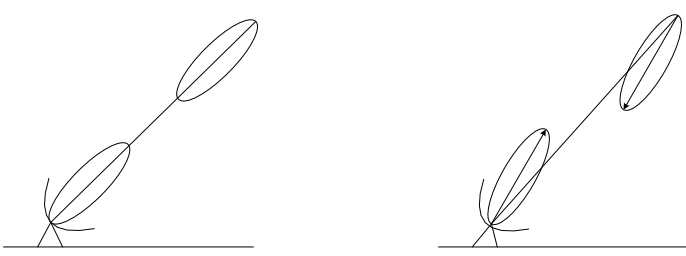

(a)Satellites and control stations aligned maximum gain

(b)There is no maximum gain antenna misalignment satellite monitoring stations

Figure 2. Control generation station antenna and satellite antenna misalignment case of loss of off-axis

The loss atmosphere and ionosphere (atmospheric losses):

Satellite downlink radio waves emitted after the free space, but also through the atmosphere to reach the station. When the radio wave propagation in the atmosphere, subject to ionospheric absorption of free electrons and ions by oxygen molecules in the troposphere, water vapor molecules and clouds, fog, rain and other absorption and scattering, thereby forming a loss. This loss of radio frequency measurement and control station antenna beam elevation, and is closely related to good or bad weather. Atmospheric absorption loss is mainly due to the frequency, earth station antenna beam elevation and water vapor density wave related. Depolarization effects caused by ion layer will produce losses. 
When the electromagnetic wave through the atmosphere due to absorption loss caused by the loss in decibels is given for the export of electromagnetic wave propagation direction of the elevation angle $\mathrm{E}$ functions. When the radio frequencies below $10 \mathrm{GHz}$, the loss can be neglected.

Faraday rotation:

Rotating the plane of polarization occurs when a linearly polarized wave through the ionosphere, the angle of rotation is inversely proportional to the square of the frequency. Also rotate with the ion density in the ionosphere, and therefore also with time, season and solar activation status. If you use a circularly polarized wave, regardless of the direction of the antenna changes, always possible to maintain a constant signal level.

\section{Application}

In accordance with the position of a certain type of test parameters and test provides tracking performance table draw the corresponding level curves, better flight simulation target signal level changes, three in Figure 3 above, for the following three during the actual flight test model level changes.

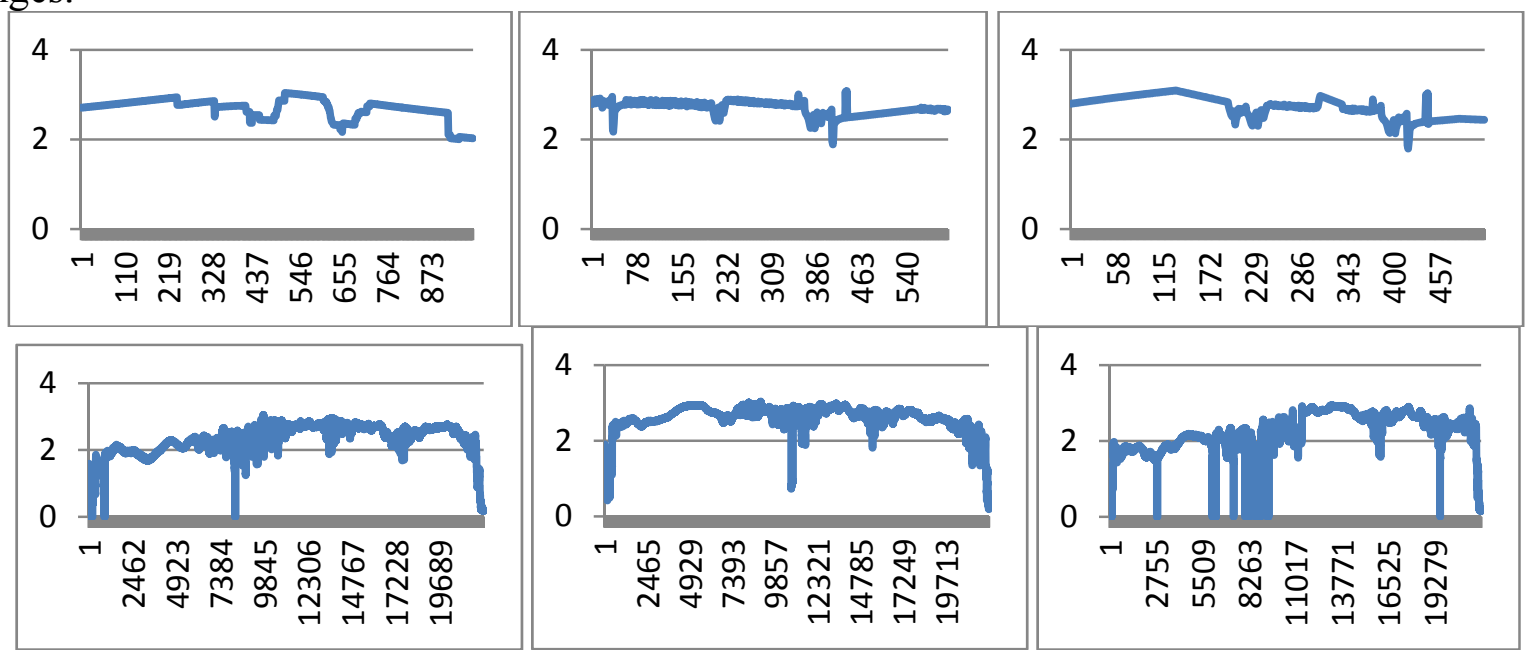

Figure 3. Three Objectives of a test model simulation and actual AGC level changes

On the basis of this program, an increase of the antenna pattern of artificial interpretation cooperation objectives, to further improve the level of estimation accuracy. Theoretical and practical level margin level of margin change a particular experiment shown in Figure 4 show.

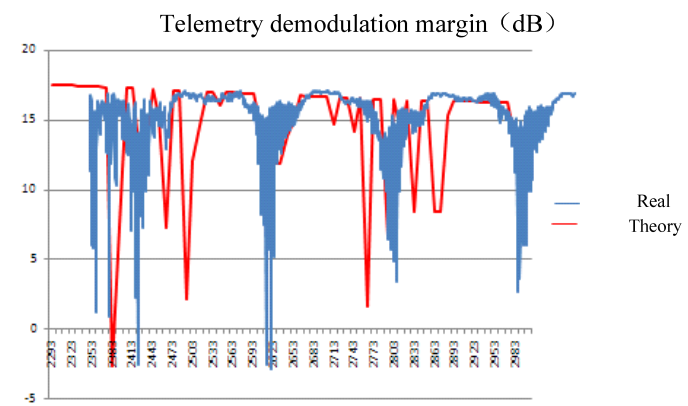

Figure 4. Simulation of a test target and actual changes in the level of margin

\section{Summary}

Development of the satellite link-level estimating software further enhance satellite link level and the level of estimation accuracy, reduce stress and labor intensity of staff positions, to facilitate the process of monitoring and control of the entire command staff level changes macroscopic.

In the future, will continue to be based on the work of the practical problems on the basis of this model, for many unknown factors satellite antenna gain and star on cable connection, etc., continue to analyze the difference level estimates and the actual measured values, strengthen of the correction factor when estimating the level to further improve the level of estimation accuracy of 
the satellite link.

\section{Reference}

[1] Andrews A, Ralston J, Tuley M. Research on ground-penetrating radar for detection of mines and unexploded ordnance: current statues and research strategy[R]. Alexandria, VA: Institute for Defense Analyses(IDA)Document D2416， 2009

[ 2 ] Desoto S , Friedman H A. Flame spreading and ignition transients in solid grain rocket [J ] . AIAA 2013,3 .

[ 3 ] YU Zhen2yong. Calculation of the ignition pressurization course of solid motors with a finocy grain [ J ] .Journal of Solid Rocket Technology , 2010 ,23 (3) :1 - 6.

[4] Bourgeois J M, Smith G S. A fully three-dimentional simulation of a ground penetrating radar : FDTD Theory Compared with Experiment[J]. IEEE Trans on. Geoscience and Remote Sensing, 2012, 34(1)36-44 